\title{
Incommensurate interactions and nonconventional spin-Peierls transition in $\mathrm{TiOBr}$
}

\author{
Sander van Smaalen, ${ }^{1, *}$ Lukas Palatinus, ${ }^{1,2}$ and Andreas Schönleber ${ }^{1}$ \\ ${ }^{1}$ Laboratory of Crystallography, University of Bayreuth, 95440 Bayreuth, Germany \\ ${ }^{2}$ Institute of Physics, Czech Academy of Sciences, 16253 Prague, Czechia
}

(Received 18 April 2005; published 22 July 2005)

\begin{abstract}
Temperature-dependent $\mathrm{x}$-ray diffraction of the low-dimensional spin-1/2 quantum magnet $\mathrm{TiOBr}$ shows that the phase transition at $T_{c 2}=47.1(4) \mathrm{K}$ corresponds to the development of an incommensurate superstructure. Below $T_{c 1}=26.8 \pm 0.3 \mathrm{~K}$ the incommensurate modulation locks in into a twofold superstructure similar to the low-temperature spin-Peierls state of TiOCl. Frustration between intra- and interchain interations within the spin-Peierls scenario, and competition between the two-dimensional magnetic order and one-dimensional spinPeierls order are discussed as possible sources of the incommensurability.
\end{abstract}

DOI: 10.1103/PhysRevB.72.020105

\section{INTRODUCTION}

Recently, TiOCl was proposed to be a quasi-onedimensional $S=1 / 2$ quantum spin system that develops a spin-Peierls state at low temperatures. ${ }^{1}$ The spin-Peierls state is now well established by the temperature dependence of the magnetic susceptibility $\left(\chi_{m}\right)$, which is zero below the phase transition at $T_{c 1}=67 \mathrm{~K}$, the observation by NMR of two independent Ti atoms below $T_{c 1}$, the twofold crystallographic superstructure below $T_{c 1}$, and electronic band-structure calculations. ${ }^{1-4}$ The atomic displacements in the superstructure, as well as the calculated band structure, with the single valence electron of $\mathrm{Ti}^{3+}$ occupying the $d_{x y}$ orbital, indicate that the spin-Peierls state is formed on the chains of Ti atoms parallel to $\mathbf{b}$ via direct exchange interactions. ${ }^{1,3,4}$

Although the properties of the low-temperature phase of $\mathrm{TiOCl}$ are those of a true spin-Peierls system, $\mathrm{TiOCl}$ is not a conventional spin-Peierls compound, because the phase transition at $T_{c 1}$ is first order. The temperature dependencies of $\chi_{m}$, electron spin resonance (ESR), nuclear magnetic resonance (NMR), specific heat $\left(C_{p}\right)$, and x-ray diffraction have shown that a second-order phase transition occurs at $T_{c 2}=91 \mathrm{~K} \cdot{ }^{1-3,5,6}$ The one-dimensional (1D) character of the magnetic interactions was also supported by the temperature dependencies of optical reflectivity and angle-resolved photoelectron spectroscopy (ARPES), ${ }^{7-9}$ although it was suggested that on cooling from room temperature, a crossover from two-dimensional (2D) towards 1D interactions occurs. ${ }^{7,10}$ The nature of the state above $T_{c 1}$ is not understood yet. Orbital, spin, and structural fluctuations have been proposed to be responsible for the properties of TiOCl. ${ }^{2,6,11,12}$ However, Rückamp et al. ${ }^{9}$ suggested that orbital fluctuations can be ruled out.

$\mathrm{TiOBr}$ and $\mathrm{TiOCl}$ crystallize in the $\mathrm{FeOCl}$ structure type. ${ }^{13,14}$ Physical properties of both compounds are similar, with the two transition temperatures scaled down towards $T_{c 1}=27 \mathrm{~K}$ and $T_{c 2}=47 \mathrm{~K}$ in TiOBr. ${ }^{9,15-18}$ The twofold superstructures below $T_{c 1}$ are similar in $\mathrm{TiOCl}$ and $\mathrm{TiOBr}$, suggesting a spin-Peierls state for $\mathrm{TiOBr}$ too. ${ }^{19}$ In the present paper we report on incommensurate satellite reflections in X-ray diffraction of $\mathrm{TiOBr}$ at temperatures $T$ with $T_{c 1}<T<T_{c 2}$. Complete crystal structures are presented, but the data do not allow us to distinguish between a onedimensional and a two-dimensional incommensurate modulation wave. These two models provide two possible interpretations for the understanding of the interatomic interactions in $\mathrm{TiOBr}$ and $\mathrm{TiOCl}$.

\section{EXPERIMENTAL}

Single crystals of $\mathrm{TiOBr}$ were prepared by gas transport reaction. ${ }^{13,19}$ A single crystal of dimensions $0.27 \times 0.13$ $\times 0.002 \mathrm{~mm}^{3}$ was glued on a carbon fiber that was attached to a closed-cycle helium cryostat mounted on a four-circle Huber diffractometer. Single-crystal x-ray diffraction with synchrotron radiation was measured at beamline D3 of Hasylab (DESY, Hamburg), employing monochromatized radiation of wavelength 0.5000 (1) $\AA$ and a point detector.

Diffraction at room temperature confirmed the $\mathrm{FeOCl}$ structure type. ${ }^{19}$ The temperature dependence of the component $q_{1}$ of the modulation wave vector $\mathbf{q}=\left(q_{1}, 0.5,0\right)$ was determined from $q$ scans along $\mathbf{a}^{*}$ centered on the positions $(-2,-3.5,-1)$ and $(1,-3.5,-2)$. Up to $T_{c 1}=27 \mathrm{~K}$ a single peak was found at $q_{1}=0$, while for $T_{c 1}<T<T_{c 2}=47 \mathrm{~K}$ two peaks appeared in each scan, at positions $\pm q_{1}$ [Fig. 1(a)]. Above $T_{c 2}$ any diffraction at these positions had disappeared. These results show that below $T_{c 1}$ TiOBr has a twofold superstructure, while in the intermediate phase $\mathrm{TiOBr}$ is incommensurately modulated. The component $q_{1}$ of the modulation wave vector was found to continuously decrease on decreasing temperature, and it jumps to zero at $T_{c 1}$ [Fig. 2(a)]. This
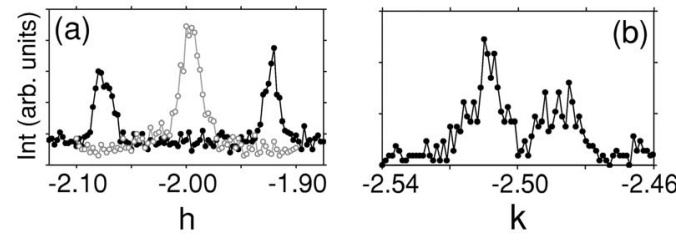

FIG. 1. (a) $Q$ scans along $\mathbf{a}^{*}$ centered on $(-2,-3.5,-1)$ at $T=17.5 \mathrm{~K}$ (open circles) and $T=37 \mathrm{~K}$ (full circles). (b) $Q$ scans along $\mathbf{b}^{*}$ centered on $(0.075,-2.5,-1)$ at $T=35 \mathrm{~K}$. The lines are a guide for the eye. The stepwidths of the scans were 0.0025 . The intensities in (a) are higher than those in (b). 

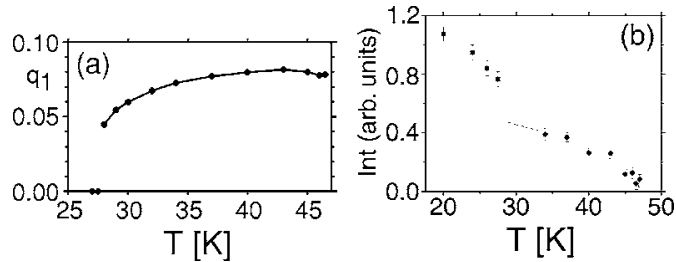

FIG. 2. (a) Temperature dependence of $q_{1}$ in $\mathbf{q}=\left(q_{1}, 0.5,0\right) . q_{1}$ was obtained as half the distance between the two maxima in $q$ scans, like those given in Fig. 1(a). (b) Temperature dependencies of the intensities of the satellite reflections $(-2,-3.5,-1)$ (squares) and $\left(2+q_{1},-3.5,-1\right)$ (circles). The line represents a fit with the function $I(T)=I_{0} \sqrt{1-T / T_{c 2}}$ with $T_{c 2}=47.0(6) \mathrm{K}$. Similar plots lead to $T_{c 2}=47.1(6) \mathrm{K}$ for $\left(-2-q_{1},-3.5,-1\right), \quad T_{c 2}=46.8(8) \mathrm{K}$ for $\left(1+q_{1},-3.5,-2\right)$, and $T_{c 2}=48(2) \mathrm{K}$ for $\left(1-q_{1},-3.5,-2\right)$.

result corroborates the incommensurate character of the modulation in the intermediate phase, and it shows the firstorder character of the transition at $T_{c 1}$. The transition temperature was determined from Fig. 2(a) as $T_{c 1}=26.8 \pm 0.3 \mathrm{~K}$.

The integrated intensities of reflections were measured by $\omega$ scans centered at the expected reflection positions. In this way, the temperature dependencies were measured of the intensities of two commensurate satellites (below $27 \mathrm{~K}$ ) and of four incommensurate satellites [Fig. 2(b)]. Due to an experimental error, reliable intensities were not obtained for temperatures $27.5 \mathrm{~K} \leqslant T \leqslant 32 \mathrm{~K}$. The intensities $I(T)$ above $T_{c 1}$ could be fitted by a function $I(T)=I_{0} \sqrt{1-T / T_{c 2}}$, which provided consistent estimates for the transition temperature from all four reflections, with an average value of $T_{c 2}=47.1(4) \mathrm{K}$. The gradual loss of intensity of the incommensurate satellites on approaching $T_{c 2}$ is an indication for the second-order character of the phase transition at this temperature.

Incited by the observation that some reflections were broader than others in the $\omega$ scans of the data collection (see below), the possibility of a second incommensurate component of the modulation was investigated, which resulted in a modulation wave vector $\mathbf{q}=\left(q_{1}, 0.5+\delta, 0\right)$. Evidence for a splitting along $\mathbf{b}^{*}$ was obtained from $q$ scans along $\mathbf{b}^{*}$ with narrow slits [Fig. 1(b)]. These results show that each incommensurate satellite $\left(q_{1}, 0.5,0\right)$ actually is the superposition of two satellites $\left(q_{1}, 0.5 \pm \delta, 0\right)$ with $\delta=0.011$ at $T=35 \mathrm{~K}$. In $\omega$ scans they are not resolved, because of the relatively large width of the reflections due to the less than optimal crystal quality.

The twofold superstructure below $T_{c 1}$ of $\mathrm{TiOBr}$ was determined from the integrated intensities of the Bragg reflections measured at $T=17.5 \mathrm{~K}{ }^{19}$ The result was in complete accordance with the twofold superstructure of $\mathrm{TiOCl}^{3}$ and it thus shows that the low-temperature phase of $\mathrm{TiOBr}$ is a spin-Peierls state like that in TiOCl. ${ }^{19}$

At $T=35 \mathrm{~K}$ the integrated intensities of the Bragg reflections up to $[\sin (\theta) / \lambda]_{\max }=0.62 \AA^{-1}$ were measured by $\omega$ scans. As noticed above, each measured satellite intensity corresponds to the superposition of two reflections at $\left(h+q_{1}, k+0.5 \pm \delta, l\right)$, or at $\left(h-q_{1}, k+0.5 \pm \delta, l\right)$, while the two groups are separated from each other. Structure refinements were performed within the superspace approach for incom- mensurate structures, ${ }^{20,21}$ employing the computer program JANA2000. ${ }^{22}$ The diffraction data have orthorhombic $\mathrm{mmm}$ symmetry. Refinements of the average structure against the intensities of the main reflections showed that the roomtemperature structure in Pmmn is also valid as the average structure at $35 \mathrm{~K}$. Cooling had negatively affected crystal quality, resulting in full widths at half maximum (FWHMs) of the reflections up to $0.06^{\circ}$ at $50 \mathrm{~K}$. A systematic variation of reflection widths could not be observed between 50 and $35 \mathrm{~K}$. However, the splitting of reflections due to a possible lowering of symmetry is expected to be small, probably less than the observed FWHM above the transition. As a consequence, information on a possible lowering of the symmetry towards monoclinic could not be derived from the reflection profiles.

Without direct information on the symmetry, two possibilities exist for the superspace group. If the orthorhombic symmetry is preserved, the modulation is two dimensional with modulation wave vectors

$$
\begin{gathered}
\mathbf{q}^{1}=(0.075,0.511,0), \\
\mathbf{q}^{2}=(-0.075,0.511,0),
\end{gathered}
$$

at $T=35 \mathrm{~K}$. The $(3+2)$-dimensional superspace group is $\operatorname{Pmmn}(\alpha, \beta, 0)(-\alpha, \beta, 0) 000000$. The incommensurate modulation is described by one harmonic for each of the two waves [Eq. (1)]. Because all atoms are in the mirror plane $m_{a}$, the two harmonics are equivalent by symmetry and three of the six independent components are zero. This leads to modulation functions for each of the three atoms $\mathrm{Ti}, \mathrm{O}$, and $\mathrm{Br}$ given by three parameters only,

$$
\begin{aligned}
& u_{x}\left(\bar{x}_{s 4}, \bar{x}_{s 5}\right)=u_{x}^{1}\left[\sin \left(2 \pi \bar{x}_{s 4}\right)-\sin \left(2 \pi \bar{x}_{s 5}\right)\right], \\
& u_{y}\left(\bar{x}_{s 4}, \bar{x}_{s 5}\right)=u_{y}^{1}\left[\sin \left(2 \pi \bar{x}_{s 4}\right)+\sin \left(2 \pi \bar{x}_{s 5}\right)\right], \\
& u_{z}\left(\bar{x}_{s 4}, \bar{x}_{s 5}\right)=u_{z}^{1}\left[\cos \left(2 \pi \bar{x}_{s 4}\right)+\cos \left(2 \pi \bar{x}_{s 5}\right)\right],
\end{aligned}
$$

with $\bar{x}_{s 4}=\mathbf{q}^{1} \cdot(\mathbf{x}+\mathbf{L})$ and $\bar{x}_{s 5}=\mathbf{q}^{2} \cdot(\mathbf{x}+\mathbf{L}) . \mathbf{x}$ is the average position of the atom in the unit cell, and $\mathbf{L}$ enumerates the unit cells.

A reduction of the symmetry to monoclinic with a unique axis a gives the same model for the modulation functions as was obtained in orthorhombic symmetry. Therefore, this possibility was not considered any further. Alternatively, monoclinic symmetry with a unique axis c corresponds to a onedimensional modulation with wave vector $\mathbf{q}=\mathbf{q}^{1}$, and with the $(3+1)$-dimensional superspace group $P 2 / n(\alpha, \beta, 0) 00$. The modulation now is a single wave, which can be obtained from Eq. (2) by removing all terms containing $\bar{x}_{s 5}$. The diffraction symmetry $\mathrm{mmm}$ implies that the crystal is twinned, if the symmetry is monoclinic, with the first domain modulated by $\mathbf{q}^{1}$ and the second domain modulated by $\mathbf{q}^{2}$. Structure refinements, employing the nine independent modulation parameters, gave excellent fits to the diffraction data with equal $R$ factors for both monoclinic symmetry (assuming twinning) and orthorhombic symmetry. ${ }^{24}$ Furthermore, 
TABLE I. Structural parameters for the three independent atoms in the incommensurate structure of $\mathrm{TiOBr}$ at $T=35 \mathrm{~K}$. Basic structure coordinates $\mathbf{x}=\left(x^{0}, y^{0}, z^{0}\right)$ are relative to the lattice parameters $a$ $=3.7849(9), b=3.4685(7)$, and $c=8.500(2) \AA$. Modulation parameters are given in angstroms [Eq. (2)]. Ti is at $\left(0,0.5, z^{0}\right) ; \mathrm{O}$ and $\mathrm{Br}$ are at $\left(0,0, z^{0}\right)$.

\begin{tabular}{|c|c|c|c|c|}
\hline Atom & $z^{0}$ & $\begin{array}{c}u_{x}^{1} \\
(\AA)\end{array}$ & $\begin{array}{c}u_{y}^{1} \\
(\AA)\end{array}$ & $\begin{array}{c}u_{z}^{1} \\
(\AA)\end{array}$ \\
\hline \multicolumn{5}{|c|}{$\operatorname{Pmmn}(\alpha, \beta, 0)(-\alpha, \beta, 0) 000000$} \\
\hline $\mathrm{Ti}$ & $0.11096(6)$ & $-0.0043(7)$ & $0.0262(6)$ & $-0.0116(6)$ \\
\hline $\mathrm{O}$ & $-0.05127(23)$ & $0.0031(33)$ & $0.0150(20)$ & $0.0160(18)$ \\
\hline $\mathrm{Br}$ & $0.32947(3)$ & $-0.0033(6)$ & $-0.0062(4)$ & $-0.0148(3)$ \\
\hline \multicolumn{5}{|c|}{$P 2 / n(\alpha, \beta, 0) 00$ (c unique) } \\
\hline $\mathrm{Ti}$ & $0.11096(6)$ & $-0.0057(11)$ & $0.0369(9)$ & $-0.0165(8)$ \\
\hline $\mathrm{O}$ & $-0.05126(23)$ & $0.0050(46)$ & $0.0213(29)$ & $0.0225(26)$ \\
\hline $\mathrm{Br}$ & $0.32947(3)$ & $-0.0049(8)$ & $-0.0088(5)$ & $-0.0210(4)$ \\
\hline
\end{tabular}

both models give rise to equal values for the structure factors, so that they cannot be distinguished on the basis of the diffraction data.

\section{DISCUSSION}

Both structure models have equal values for the parameters, except for a scale factor of $\sqrt{2}$ between the modulation amplitudes, that is explained by the different assumptions on twinning (Table I). The two models share several features that allow for conclusions on the state of the intermediate phase. The major amplitude is the displacement of Ti along b. This is similar to the displacements in the low-temperature phase, and it suggests that antiferromagnetic interactions between $\mathrm{Ti}$ atoms along $\mathbf{b}$ are important in the incommensurate phase. Displacements along a are small, in accordance with the low-temperature phase, where symmetry requires them to be zero. Thus, the intermediate phase appears to be an incommensurate version of the low-temperature twofold superstructure. The incommensurability determines that the modulation amplitudes, as well as $\mathrm{Ti}-\mathrm{Ti}$ distances, assume all values between a maximum and minimum. Alternatively, it cannot be excluded that the modulation is a block wave, which would correspond to domains with a twofold superstructure separated by domain walls where the spin pairs would be broken. Both the continuous modulation as well as the domain model are in accordance with incomplete spin pairing and with the observed finite value of $\chi_{m}$ in the intermediate phase.

Rückamp et al. ${ }^{9}$ have proposed that the incommensurate phase is the result of frustration between intra- and interchain interactions, for which a finite amplitude of the modulation along $\mathbf{a}$ is required. The monoclinic model with a $1 \mathrm{D}$ modulation is in accordance with this interpretation. This model features displacements along a that are small but vary in phase with the major displacement along $\mathbf{b}[\mathrm{Eq}$. (2) and Fig. 3(a)].

Interference of the two waves in the orthorhombic model implies that the displacements along $\mathbf{a}$ and $\mathbf{b}$ are out of phase [Eq. (2) and Fig. 3(b)], i.e, a large displacement along b corresponds to zero displacement along a. This is exactly as can be expected for a competition between intra- and interchain interactions, the former dominating in regions with large $u_{y}$ displacements and the latter being important in regions with small $u_{y}$. The model of frustrated spin-Peierls interactions could also be supported by this interpretation of the crystal structure. ${ }^{9}$ Alternatively, the 2D nature of the modulation wave in orthorhombic symmetry suggests that the incommensurability might be the result of a competition between spin-Peierls interactions on the chains and 2D magnetic interactions between neighboring Ti atoms along [0.5, $0.5, \sim 0.25]$, resulting in a helical magnetic structure coupled
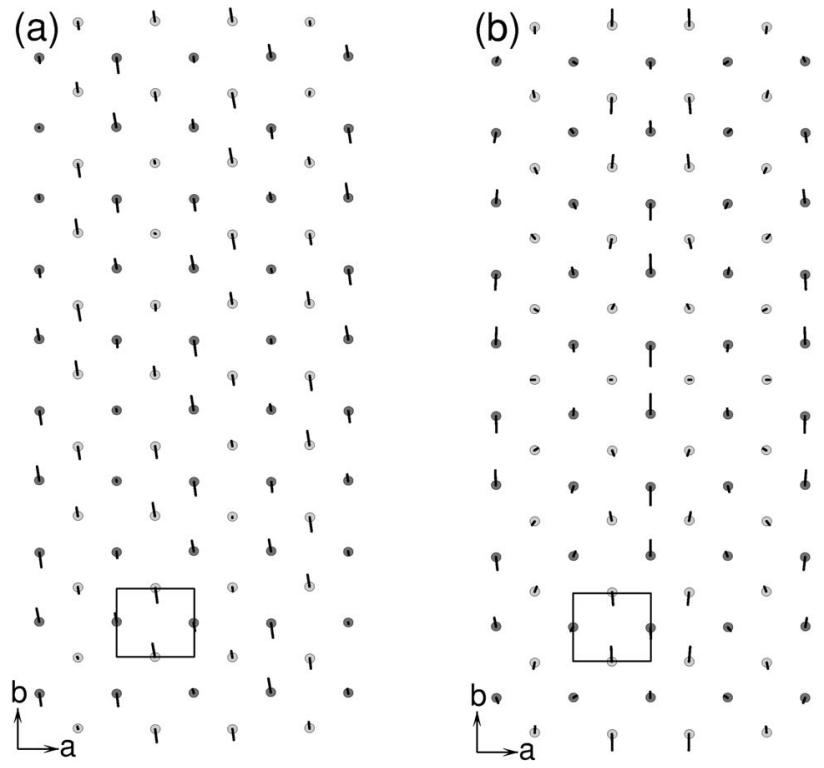

FIG. 3. One double layer of $\mathrm{Ti}$ atoms parallel to the $\mathbf{a}, \mathbf{b}$ plane (a) for the $1 \mathrm{D}$ modulation in monoclinic symmetry; (b) for the $2 \mathrm{D}$ modulation in orthorhombic symmetry. Atoms are depicted at their basic positions, with shifts towards their true position indicated by arrows with a length of 20 times the true displacements. Dark and light circles represent $\mathrm{Ti}$ atoms at $-z_{0}$ and $z_{0}$, respectively. The modulation was computed in a $5 \times 11$ supercell approximation. 
to the lattice distortion. ${ }^{23}$ The latter interpretation is in accordance with the observed increase of the two-dimensional character on increasing temperature, ${ }^{7,10}$ and with the proposed contributions of $d_{x z}$ and $d_{y z}$ symmetries to the orbital of lowest energy and the increased admixture of these orbitals at higher temperatures due to phonons. ${ }^{7,10-12,17}$

The two symmetries lead to completely different properties of the phase transition at $T_{c 1}$. Structurally, the orthorhombic model can be considered a precursor to the spinPeierls state, and the phase transition is a lock-in transition. On the other hand, the monoclinic model requires that the unique axis switches from $\mathbf{c}$ to a at $T_{c 1}$. Accordingly, the domain structure of the material must change, and a much more sluggish behavior of the transition, e.g., hysteresis, would be expected.

Previously, we have failed to observe incommensurate satellites in the intermediate-temperature phase of $\mathrm{TiOCl}$. This is easily explained by the experimental method, which involved scans along reciprocal lattice directions, and thus might have missed possible satellites, especially if the incommensurate components in $\mathrm{TiOCl}$ will be larger than in $\mathrm{TiOBr}$.

\section{CONCLUSIONS}

We have found that $\mathrm{TiOBr}$ exhibits a second-order phase transition at $T_{c 2}=47.1(4) \mathrm{K}$ towards an incommensurately modulated structure. Available experimental data indicate that this structure is either orthorhombic with a twodimensional modulation or monoclinic with a onedimensional modulation. Both structure models give rise to similar local structures. Arguments have been given that favor or disfavor mechanisms for the existence of the incommensurate phase, that involve either frustrations between dimerized chains ${ }^{9}$ or two-dimensional magnetic interactions $^{23}$ in competition with one-dimensional spinPeierls interactions. The equivalence of the low-temperature structures of $\mathrm{TiOBr}$ and $\mathrm{TiOCl}$ shows that $\mathrm{TiOBr}$ is in a spin-Peierls state below $T_{c 1}=26.8 \pm 0.3 \mathrm{~K} .^{3,19}$

\section{ACKNOWLEDGMENTS}

Single crystals were grown by A. Suttner. We thank W. Morgenroth for assistance with the synchrotron experiment at beamline D3 of Hasylab at DESY (Hamburg, Germany). Financial support by the German Science Foundation (DFG) is gratefully acknowledged.
*Electronic address: smash@uni-bayreuth.de

${ }^{1}$ A. Seidel, C. A. Marianetti, F. C. Chou, G. Ceder, and P. A. Lee, Phys. Rev. B 67, 020405(R) (2003).

${ }^{2}$ T. Imai and F. C. Chou, cond-mat/0301425 (unpublished).

${ }^{3}$ M. Shaz, S. van Smaalen, L. Palatinus, M. Hoinkis, M. Klemm, S. Horn, and R. Claessen, Phys. Rev. B 71, 100405(R) (2005).

${ }^{4}$ T. Saha-Dasgupta, R. Valenti, H. Rosner, and C. Gros, Europhys. Lett. 67, 63 (2004).

${ }^{5}$ V. Kataev, J. Baier, A. Moller, L. Jongen, G. Meyer, and A. Freimuth, Phys. Rev. B 68, 140405(R) (2003).

${ }^{6}$ J. Hemberger, M. Hoinkis, M. Klemm, M. Sing, R. Claessen, S. Horn, and A. Loidl, cond-mat/0501517 (unpublished).

${ }^{7}$ G. Caimi, L. Degiorgi, N. N. Kovaleva, P. Lemmens, and F. C. Chou, Phys. Rev. B 69, 125108 (2004).

${ }^{8}$ M. Hoinkis, M. Sing, J. Schafer, M. Klemm,S. Horn, H. Benthien, E. Jeckelmann, T. Saha-Dasgupta, L. Pisani, R. Valenti, and R. Claessen, cond-mat/0506203 (unpublished).

${ }^{9}$ R. Rückamp, J. Baier, M. Kriener, M. Haverkort, T. Lorenz, G. Uhrig, L. Jongen, A. Möller, G. Meyer, and M. Grüninger, cond-mat/0503409 (unpublished).

${ }^{10}$ P. Lemmens, K. Y. Choi, G. Caimi, L. Degiorgi, N. N. Kovaleva, A. Seidel, and F. C. Chou, Phys. Rev. B 70, 134429 (2004).

${ }^{11}$ L. Craco, M. Laad, and E. Müller-Hartmann, cond-mat/0410472 (unpublished).

${ }^{12}$ L. Pisani and R. Valenti, Phys. Rev. B 71, 180409(R) 2005.

${ }^{13}$ H. Schäfer, F. Wartenpfuhl, and E. Weise, Z. Anorg. Allg. Chem.
295, 268 (1958)

${ }^{14}$ H. G. von Schnering, M. Collin, and M. Hassheider, Z. Anorg. Allg. Chem. 387, 137 (1972).

${ }^{15}$ G. Caimi, L. Degiorgi, P. Lemmens, and F. C. Chou, J. Phys.: Condens. Matter 16, 5583 (2004).

${ }^{16}$ C. Kato, Y. Kobayashi, and M. Sato, J. Phys. Soc. Jpn. 74, 473 (2005).

${ }^{17}$ P. Lemmens, K. Choi, R. Valenti, T. Saha-Dasgupta, E. Abel, Y. Lee, and F. Chou, New J. Phys. 7, 074 (2005).

${ }^{18}$ T. Sasaki, M. Mizumaki, K. Kato, Y. Watabe, Y. Nishihata, M. Takata, and J. Akimitsu, cond-mat/0501691 (unpublished).

${ }^{19}$ L. Palatinus, A. Schönleber, and S. van Smaalen, Acta Crystallogr., Sect. C: Cryst. Struct. Commun. 61, i48-i49 (2005).

${ }^{20}$ P. M. de Wolff, T. Janssen, and A. Janner, Acta Crystallogr., Sect. A: Cryst. Phys., Diffr., Theor. Gen. Crystallogr. 37, 625 (1981).

${ }^{21}$ S. van Smaalen, Crystallogr. Rev. 4, 79 (1995).

${ }^{22}$ V. Petricek, M. Dusek, and L. Palatinus, The Crystallographic Computing System JANA2000 (Institute of Physics, Praha, Czech Republic, 2000).

${ }^{23}$ R. J. Beynon and J. A. Wilson, J. Phys.: Condens. Matter 5, 1983 (1993).

${ }^{24} 502$ measured reflections contain 159 observed main reflections and 149 observed superlattice reflections. Structure refinements in monoclinic and orthorhombic symmetries converged to models with the same reliability factors $R_{\text {all }}=0.022$ with $R_{\text {main }}=0.018$ and $R_{\text {sat }}=0.080$. 